\title{
Specific genomic alterations and prognostic analysis of perihilar cholangiocarcinoma and distal cholangiocarcinoma
}

\author{
Yuanwen Zheng ${ }^{1,2}$, Yejun Qin ${ }^{3}$, Wei Gong ${ }^{1}$, Hongguang $\mathrm{Li}^{1}$, Bin $\mathrm{Li}^{4}$, Yu Wang ${ }^{5}$, Baoting Chao ${ }^{2}$, \\ Shulei Zhao ${ }^{4}$, Luguang Liu ${ }^{6}$, Shuzhan Yao ${ }^{7}$, Junping Shi ${ }^{8}$, Xiaoliang Shi ${ }^{8}$, Kai Wang ${ }^{8}$, Shifeng Xu ${ }^{1,9} \wedge$ \\ ${ }^{1}$ Department of Hepatobiliary Surgery, Shandong Provincial Hospital Affiliated to Shandong First Medical University, Jinan, China; ${ }^{2}$ School of \\ Clinical Medicine, Cheeloo College of Medicine, Shandong University, Jinan, China; ${ }^{3}$ Department of Pathology, Shandong Provincial Hospital \\ Affiliated to Shandong First Medical University, Jinan, China; ${ }^{4}$ Department of Gastroenterology, Shandong Provincial Hospital Affiliated to \\ Shandong First Medical University, Jinan, China; ${ }^{5}$ Tumor Research and Therapy Center, Shandong Provincial Hospital Affiliated to Shandong First \\ Medical University, Jinan, China; ${ }^{6}$ Department of Gastrointestinal Surgery, Cancer Hospital Affiliated to Shandong First Medical University, Jinan, \\ China; ${ }^{7}$ Department of Medical Imaging, Shandong Provincial Hospital Affiliated to Shandong First Medical University, Jinan, China; ${ }^{8}$ Shanghai \\ OrigiMed Co., Ltd., Shanghai, China; ${ }^{9}$ Department of Hepatobiliary Surgery, Shandong Provincial Hospital Affiliated to Shandong University, \\ Jinan, China \\ Contributions: (I) Conception and design: S Xu, Y Zheng; (II) Administrative support: S Xu, Y Wang, K Wang; (III) Provision of study materials \\ or patients: S Xu, Y Zheng, Y Qin, W Gong, S Zhao, B Chao; (IV) Collection and assembly of data: Y Zheng, H Li, B Li, L Liu, S Yao; (V) Data \\ analysis and interpretation: Y Zheng, S Xu, J Shi, X Shi; (VI) Manuscript writing: All authors; (VII) Final approval of manuscript: All authors. \\ Correspondence to: Prof. Shifeng Xu. Department of Hepatobiliary Surgery, Shandong Provincial Hospital Affiliated to Shandong First Medical \\ University, Jinan 250021, China. Email: shifengxu2008@163.com.
}

Background: Cholangiocarcinoma (CCA), which consists of intrahepatic CCA (iCCA), perihilar CCA (pCCA), and distal CCA (dCCA), is an aggressive malignancy worldwide. PCCA and dCCA are often classified as extrahepatic CCA (exCCA). However, the differences in mutational characteristics between pCCA and dCCA remain unclear.

Methods: Deep sequencing targeting of 450 cancer genes was performed for genomic alteration detection. The tumor mutational burden (TMB) was measured by an algorithm developed in-house. Correlation analysis was conducted using Fisher's exact test.

Results: FGFR2 and ERBB2 mutations mainly occurred in iCCA and exCCA, respectively. In exCCA, the frequencies of PIK3CA, FAT4, KDM6A, MDM2, and TCF7L2 mutations were significantly higher in pCCA compared to dCCA, while the frequencies of TP53 and KRAS mutations were markedly lower in pCCA than those in dCCA. The prognosis-related mutations were different among the CCA subtypes. NF1 mutation was associated with short disease-free survival (DFS) and overall survival (OS), and ERBB2 mutation was associated with short DFS in dCCA patients. Meanwhile, MAP2K4 mutation was associated with long DFS and OS, and TERT mutation was associated with short DFS in pCCA. A series of mutations in genes, including ARID1A, ARID2, SMAD4, TERT, TP53, and KRAS, were found to be associated with the TMB.

Conclusions: In this study, we investigated the comprehensive genomic characterizations of CCA patients, identified the significant alterations in each subtype, and identified potential biomarkers for prognosis prediction. These results provide molecular evidence for the heterogeneity of CCA subtypes and evidence for further precision targeted therapy of CCA patients.

Keywords: CCA subtype; biomarker; tumor mutational burden (TMB); next-generation sequencing (NGS); prognosis

Submitted Oct 25, 2021. Accepted for publication Dec 16, 2021.

doi: 10.21037/jgo-21-776

View this article at: https://dx.doi.org/10.21037/jgo-21-776

$\wedge$ ORCID: 0000-0002-0590-3642. 


\section{Introduction}

Cholangiocarcinoma (CCA) is an invasive malignancy tumor derived from bile duct epithelial cells. Early biliary tract cancer (BTC) has no clearly symptoms, and only a few BTC patients are considered as surgical resection at the initial diagnosis (1). BTCs originating in the bile ducts can be classified as intrahepatic (iCCAs), perihilar (pCCAs; Klatskin tumors), or distal cholangiocarcinoma (dCCAs) according to their anatomical location, each with distinct epidemiological and molecular pathological processes (2). PCCA and dCCA are bounded by cystic duct and common hepatic duct. Patients with dCCA often find gallbladder dilatation, intrahepatic bile duct dilatation, and extrahepatic bile duct dilatation, while patients with pCCA often show perihepatic bile duct dilatation, normal size of common bile duct and possible contraction of gallbladder. Both pCCA and dCCA are normally classified as extrahepatic CCA (exCCA) (2). The risk factors of iCCA and exCCA are different. For example, Cirrhosis, hepatitis B and $\mathrm{C}$ viruses, inflammatory bowel disease and type 2 diabetes mellitus were found to be more strongly association with iCCA, and choledochal cyst, choledocholithiasis, cholelithiasis and smoking were more at risk in exCCA (3). The difference in risk factors between pCCA and dCCA has rarely been reported. To date, surgery is the preferred treatment candidate for CCA subtypes. However, only a few CCA patients in early stage may receive surgical resection (4). As previously reported, surgical resection is associated with disease-free survival (DFS) in patients with iCCA (5). A high recurrence risk and poor survival outcomes are associated with iCCA surgery and liver transplantation (6). Currently, combination chemotherapy is the first-line treatment for advanced-stage CCA patients that are not suitable for surgical or locoregional options (7). Valle et al. reported that the median overall survival (OS) of gemcitabine combined with cisplatin was longer than that of gemcitabine alone (11.7 months versus 8.1 months, respectively) (8). For patients who progressed from first-line gemcitabine-based chemotherapy, second line and above antitumor treatments are limited.

Importantly, as one of the most heterogeneous tumors in terms of molecular features, survival prognosis and therapeutic responses are varied in BTC patients. Recently, more and more molecularly targeted therapies have been investigated in early CCA clinical trials (9). Comprehensive whole-exome and transcriptome analysis based on large BTC cohort had revealed potentially targetable genetic driver alterations (10). The specific mutations include IDH1, MCL1, PBRM1, FGFR2, and FGFR 3/4/19 in iCCA, and $F B X W 7$, ERBB2, and RBM10 in exCCA $(10,11)$. Previous studies have also shown the genomic heterogeneity of CCA subtypes, potentially affecting future therapy trials (12). Waseem et al. reported that the mean survival of pCCA is lower than that of dCCA, but is similar to iCCA (13). However, few studies isolated pCCA and focused on its genomic characteristics.

To identify the underlying genomic targets with clinical translational significance, we systemically analyzed 270 CCA samples from Chinese populations. We comprehensively analyzed the genomic mutational profiling and distinguished the molecular features between pCCA and dCCA. We present the following article in accordance with the REMARK reporting checklist (available at https:// dx.doi.org/10.21037/jgo-21-776).

\section{Methods}

\section{Patient selection and review}

A total of 270 CCA patients who received surgical treatment between 2014 and 2019 were enrolled. The study was conducted according to the guidelines of the Declaration of Helsinki (as revised in 2013). Written informed consent for tumor genomics profiling was obtained from each patient. The study protocol was approved by the Institutional Ethics Review Committee at Shandong Provincial Hospital (ethics approval number: LCYJ: No. 2019-081). Informed consent was obtained from all subjects involved in the study. The clinical data and follow up information were obtained from the electronic medical record or by telephone inquiry.

\section{Next-generation sequencing (NGS)}

Genomic alterations were detected by using the YuanSu 450 panel in the OrigiMed, a College of American Pathologists (CAP)-accredited and Clinical Laboratory Improvement Amendments (CLIA)-certified laboratory (Shanghai OrigiMed Co., Ltd, Shanghai, China),. At least $50 \mathrm{ng}$ of cancer tissue DNA was extracted from each $40 \mathrm{~mm}^{3}$ formalin-fixed, paraffin embedded (FFPE) tumor sample using a DNA extraction kit (QIAamp DNA FFPE Tissue Kit, Qiagen, Hilden, Germany) according to the manufacturer's protocol. YuanSu 450 panel cover the all coding exons of 450 tumor-related genes and the selected introns of 39 commonly rearranged genes in solid tumors. 
The genes were captured and sequenced, with a mean coverage of $900 \times$ for FFPE samples and $300 \times$ for matched paracancerous samples, using Illumina NextSeq-500 (Illumina Inc., San Diego, CA). Genomic alterations including single nucleotide variants (SNVs), short and long insertions/deletions (Indels), copy number variations, and structural variants of gene rearrangement/fusion were further analyzed.

\section{Tumor mutational burden (TMB) calculations}

The TMB was caculated by counting the somatic mutations, including SNVs and Indels, per megabase of the sequence examined for each patient. Driver mutations and known germline alterations were not counted.

\section{Statistical analysis}

Data analyses were performed with SPSS statistical software (version 22.0; IBM, USA). Comparisons between the groups were performed using the $\chi^{2}$ test when appropriate. A multinomial logistic regression model was used to estimate the odds ratio. Kaplan-Meier curves were applied to present the survival probability of different patients. $\mathrm{P}<0.05$ was considered statistically significant.

\section{Results}

\section{Samples and patient clinical characteristics}

A total of 270 CCA patients, including 92 iCCA, 70 pCCA, and $108 \mathrm{dCCA}$ patients were enrolled in this study. The median age of patients was 61 years old (range, 18-79 years). Among them, 30.7\% (83/270) were male and $69.3 \%$ $(187 / 270)$ were female. A total of $55.2 \%(149 / 270)$ were well to moderately differentiated, $78.9 \%$ (213/270) were N0 status, and 4.4\% (12/270) possessed a confirmed cancerrelated family history (Table 1). Although preoperative evaluation showed that all tumors were surgically resected, 25.6\% (69/270) were R1/R2 resections. According to the American Joint Committee on cancer (AJCC) $8^{\text {th }}$ edition, postoperative evaluation showed that $41.3 \%$ of iCCA patients were stage III/IV, while $25.7 \%$ and $12.0 \%$ of pCCA and dCCA patients were stage III/IV, respectively. Statistical analysis demonstrated the significant association between tumor stage and CCA subtypes $(\mathrm{P}<0.001)$. Most of CCA patients were hepatitis $\mathrm{B}$ virus/hepatitis $\mathrm{C}$ virus (HBV/ $\mathrm{HCV}$ ) negative $(87.8 \%, 237 / 270)$, and the $\mathrm{HBV} / \mathrm{HCV}$ positivity rate was significantly higher in iCCA than that in exCCA $(19.6 \%$ vs. $4.8 \% ; \mathrm{P}<0.001)$ (Table 1).

\section{Characterization of genomic alterations}

A total of 1,711 mutants from 395 genes were identified in 270 CCA samples (6.3 mutations/sample), which included 943 somatic SNVs or small Indels (Substitutions/Indels), 441 truncations, 258 gene amplifications, 46 fusions/ rearrangements, and 23 gene homozygous deletions (Table S1). No mutations were detected in eight patients in the 450-gene panel. The most commonly mutated genes of Chinese CCA patients were TP53 (56\%, 151/270), followed by KRAS (32\%, 86/270), SMAD4 (16\%, 44/270), CDKN2A (16\%, 42/270), ARID1A (15\%, 41/270), ARID2 (12\%, $32 / 270)$, and TERT (12\%, 32/270) (Figure 1). Functional pathways of the cell cycle $(66 \%, 178 / 270), M A P K(50 \%$, 134/270), PI3K (24\%, 66/270), and HRD (16\%, 42/270) were frequently altered in CCA patients (Figure S1).

\section{Different mutational characteristics of iCCA and exCCA}

It is well known that exCCA and iCCA exhibit different molecular mutation characteristics. In this study, we found that BAP1, PBRM1, and FGFR2 mutations occurred frequently in iCCA, while ERBB2 and SMAD4 mutations occurred frequently in exCCA. Statistical analysis showed that BAP1, PBRM1, and FGFR2 mutations were significantly associated with iCCA $\left(\mathrm{P}=7.47 \times 10^{-5}, \mathrm{P}=7347 \times 10^{-5}\right.$, and $\mathrm{P}=0.0002$, respectively), and $E R B B 2$ and $S M A D 4$ mutations were significantly associated with exCCA $(\mathrm{P}=0.0088$ and $\mathrm{P}=0.037$, respectively). Nine (9.8\%) iCCA patients harbored FGFR2 fusion in this cohort. FGFR2 fusion occurred more commonly in females ( $17 \%$ vs. $5 \% ; \mathrm{P}=0.08)$. Although TP53 mutations occurred frequently in both iCCA and exCCA, they were markedly more frequent in exCCA than in iCCA. Meanwhile, it should be noted that IDH1 mutations (6.5\% vs. $0 \% ; \mathrm{P}=0.0015)$ occurred specifically in iCCA (Figure 2A).

\section{Differentiation of molecular characteristics in exCCA subtypes}

Although pCCA and dCCA are classified as exCCA, their molecular characteristics were different. TERT, PIK3CA, $M D M 2, F A T 4$, and KDM6A mutations were more prevalent in pCCA than in dCCA (Figure S2). Among these genes, the mutation frequencies of TERT and PIK3CA in pCCA 
Table 1 Clinicopathological features of Chinese CCA patients

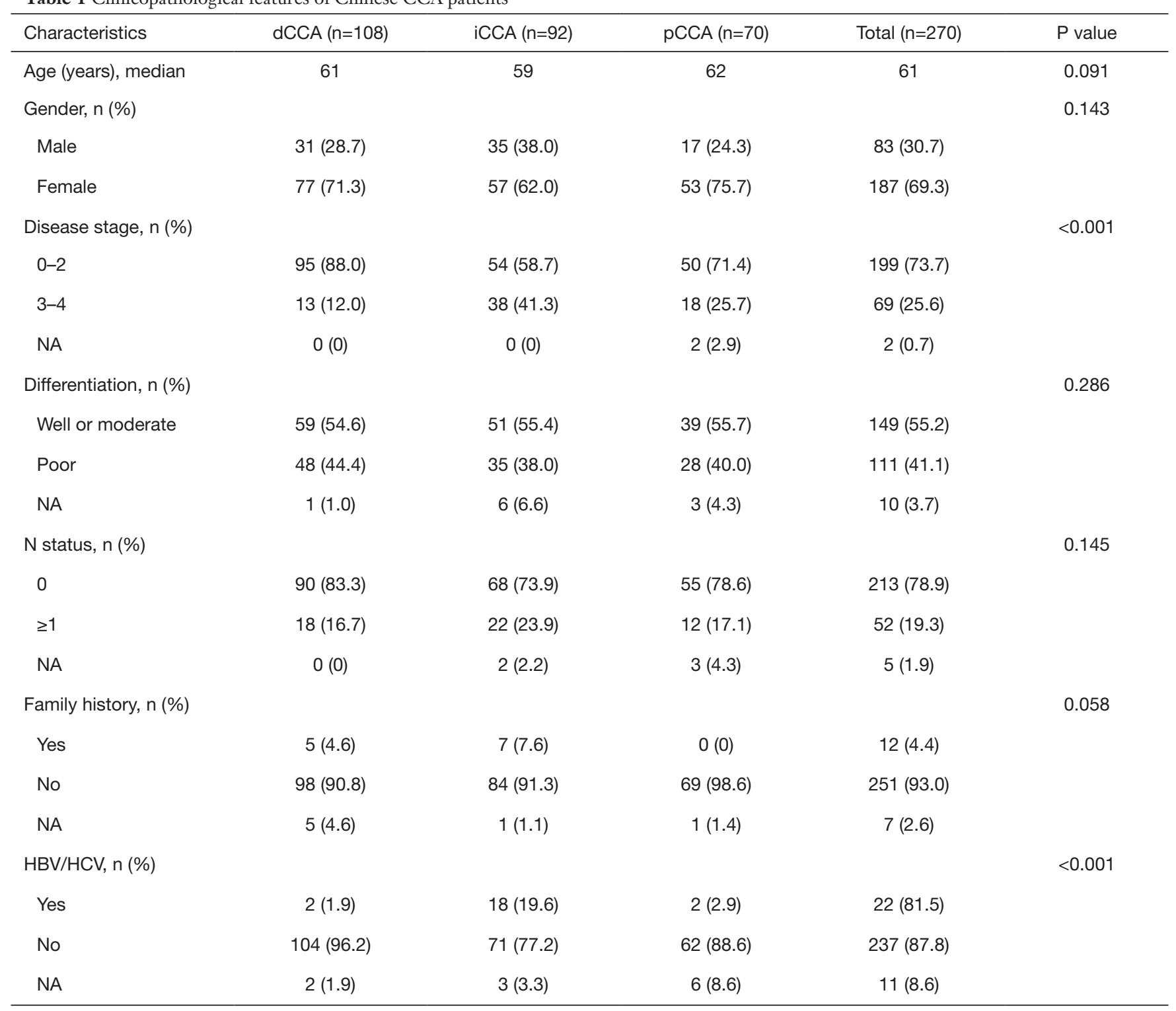

CCA, cholangiocarcinoma; dCCA, distal CCA; iCCA, intrahepatic CCA; pCCA, perihilar CCA; NA, not available.

were similar to those of iCCA, while the mutations of $M D M 2, F A T 4$, and KDM6A were specifically prevalent in pCCA (Figure S2). Statistical analysis showed notably higher frequencies of PIK3CA, FAT4, KDM6A, MDM2, and TCF 7L2 mutations in pCCA than in DCCA, and significantly lower frequencies of TP53 and KRAS mutations in pCCA than in dCCA (Figure 2B).

\section{$T M B$}

To investigate the potential guidance in the treatment of
CCA, we identified the TMB value of this cohort. The median TMB was 3.1 muts/Mb (range, 0-50.2 muts/Mb). There was no significant difference in the distribution of the TMB among the different tumor subtypes. The $80 \%$ TMB of CCA was 6.2 muts/Mb; therefore, TMB values higher than 6.2 muts/ $\mathrm{Mb}$ were considered as a high TMB (TMB-H), and TMB values lower than 6.2 muts/Mb were considered as a low TMB (TMB-L).

We investigated the correlation between the most commonly mutated genes and the TMB. The results showed that ARID1A ( $\mathrm{P}=0.025)$, ARID2 ( $\mathrm{P}=0.005)$, SMAD4 


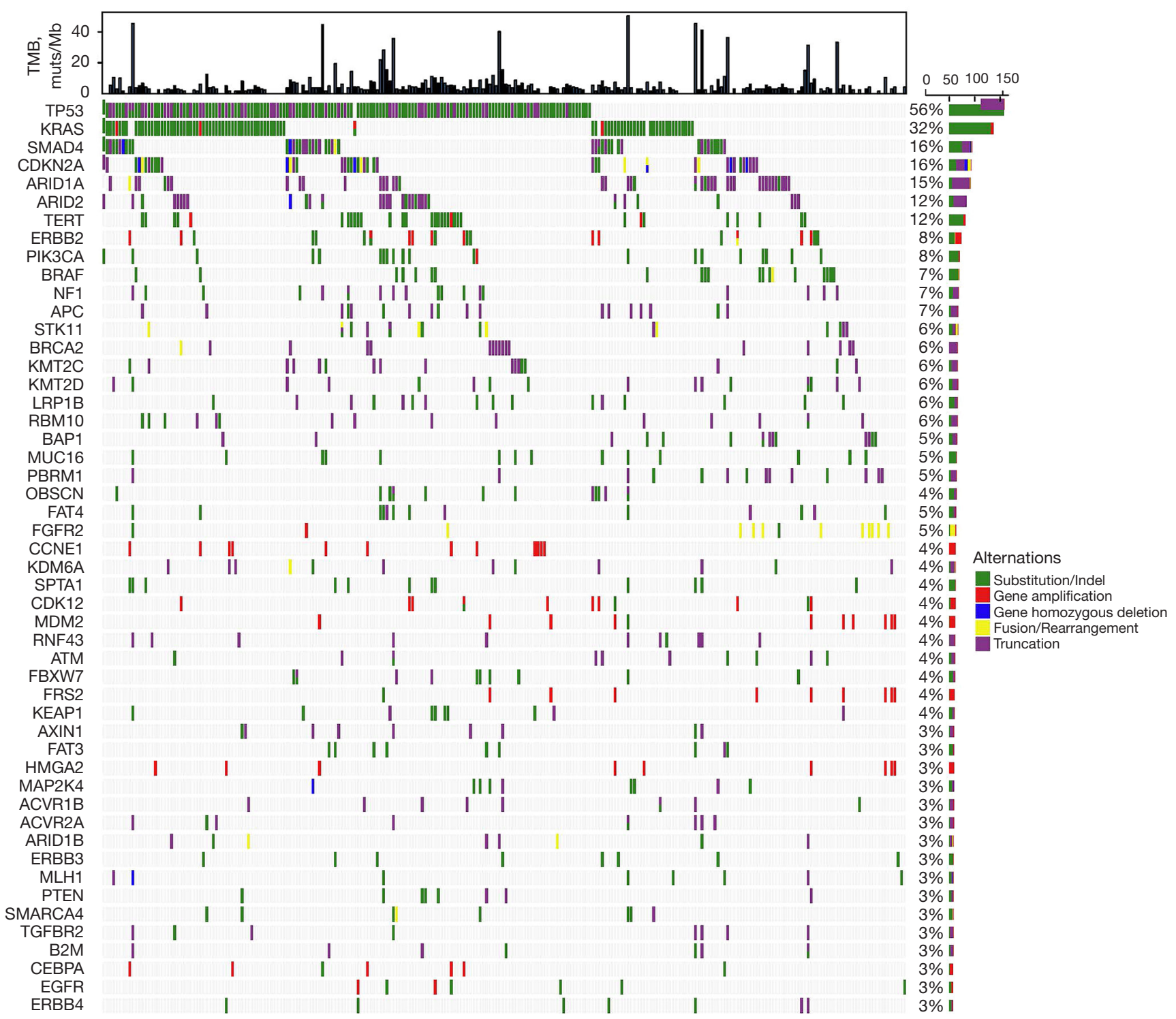

Figure 1 Most common genomic alterations of 270 CCA samples. Each column represents a case sample. Partial mutated genes of samples are listed on the left side. The top bar graph represents the TMB of each sample, and the right bar graph represents the mutational frequency of corresponding mutated gene. Different colors show mutational types at the right side of panel. CCA, cholangiocarcinoma; TMB, tumor mutational burden.

( $\mathrm{P}=0.046)$, TERT $(\mathrm{P}=0.004)$, and TP53 ( $\mathrm{P}=0.001)$ mutations were significantly associated with TMB-H, while the KRAS $(\mathrm{P}=0.001)$ mutations were notably associated with TMB-L (Figure 3).

\section{Analysis of DFS and OS in patients}

To exclude the influence of advanced tumor on DFS and OS, 199 early CCA patients with tumor stage I/II were selected for further study. The DFS and OS data from 143 and 135 patients, respectively, were collected for further analysis. Of the 143 patients with effective DFS information, there were $67 \mathrm{dCCAs,} 36 \mathrm{iCCAs,}$ and 40 pCCAs; the median DFS was 11 months (range, 1-53 months), 4.5 months (range, 2-31 months), and 11 months (range, 2-72 months), respectively. Statistical 

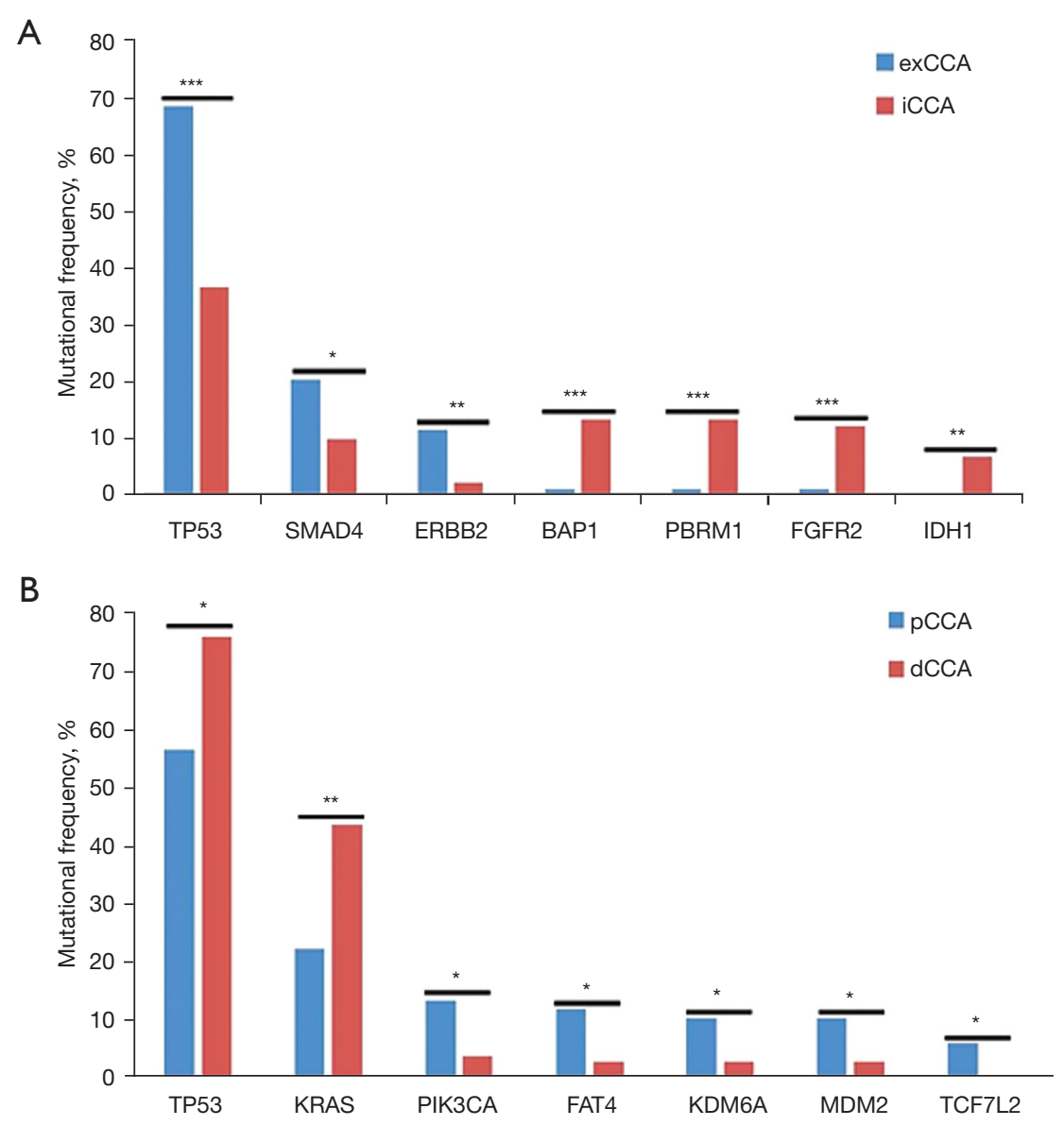

Figure 2 The significantly different mutated genes between iCCA and exCCA (A) and between pCCA and dCCA (B). The X-axis represents the mutated gene and the $\mathrm{Y}$-axis represents the mutational frequency of each gene. Blue represents the exCCA group, while red represents the iCCA group. *, $\mathrm{P}<0.05$; **, $\mathrm{P}<0.01$; ***, $\mathrm{P}<0.001$. iCCA, intrahepatic cholangiocarcinoma; exCCA, extrahepatic cholangiocarcinoma; pCCA, perihilar cholangiocarcinoma; dCCA, distal cholangiocarcinoma.

analysis showed significantly longer DFS of dCCA and pCCA compared to iCCA $(\mathrm{P}=0.002)$ (Figure $4 A)$. Of the 135 patients with effective OS information, there were 60 dCCA, 33 iCCA, and 42 pCCA; the median OS was 17.5, 9, and 16 months, respectively. Statistical analysis showed that the survival rate of dCCA was significantly higher than that of iCCA ( $\mathrm{P}=0.036)$ (Figure 4B). No significant differences were detected between pCCA and dCCA patients for both DFS and OS.

We selected genes with $>10 \%$ mutation frequency for further correlation analysis. For CCA patients, the NF1 mutation was associated with a short DFS and OS, while the MAP2K4 mutation was associated with a long DFS (Figure $5 A$ ). For CCA subtypes, we found that NF1 mutations were associated with a short DFS and OS, and ERBB2 mutations were associated with short DFS in
dCCA patients (Figure 5B). Also, MAP2K4 mutations were associated with a long DFS and OS, and TERT mutations were associated with a short DFS in pCCA (Figure 5C). Furthermore, RBM10 mutations were associated with a short DFS and OS, and KRAS mutations were associated with a short DFS in iCCA (Figure 5D). Interestingly, no mutated genes associated with DFS or OS of two or more CCA subtypes were detected.

\section{Discussion}

CCA is a malignant tumor originating from bile duct epithelium, which has a complex etiology and atypical clinical characteristics. Surgical incision is an effective therapy for early CCA. However, clear classification of CCA subtypes is helpful for further treatment. With the 

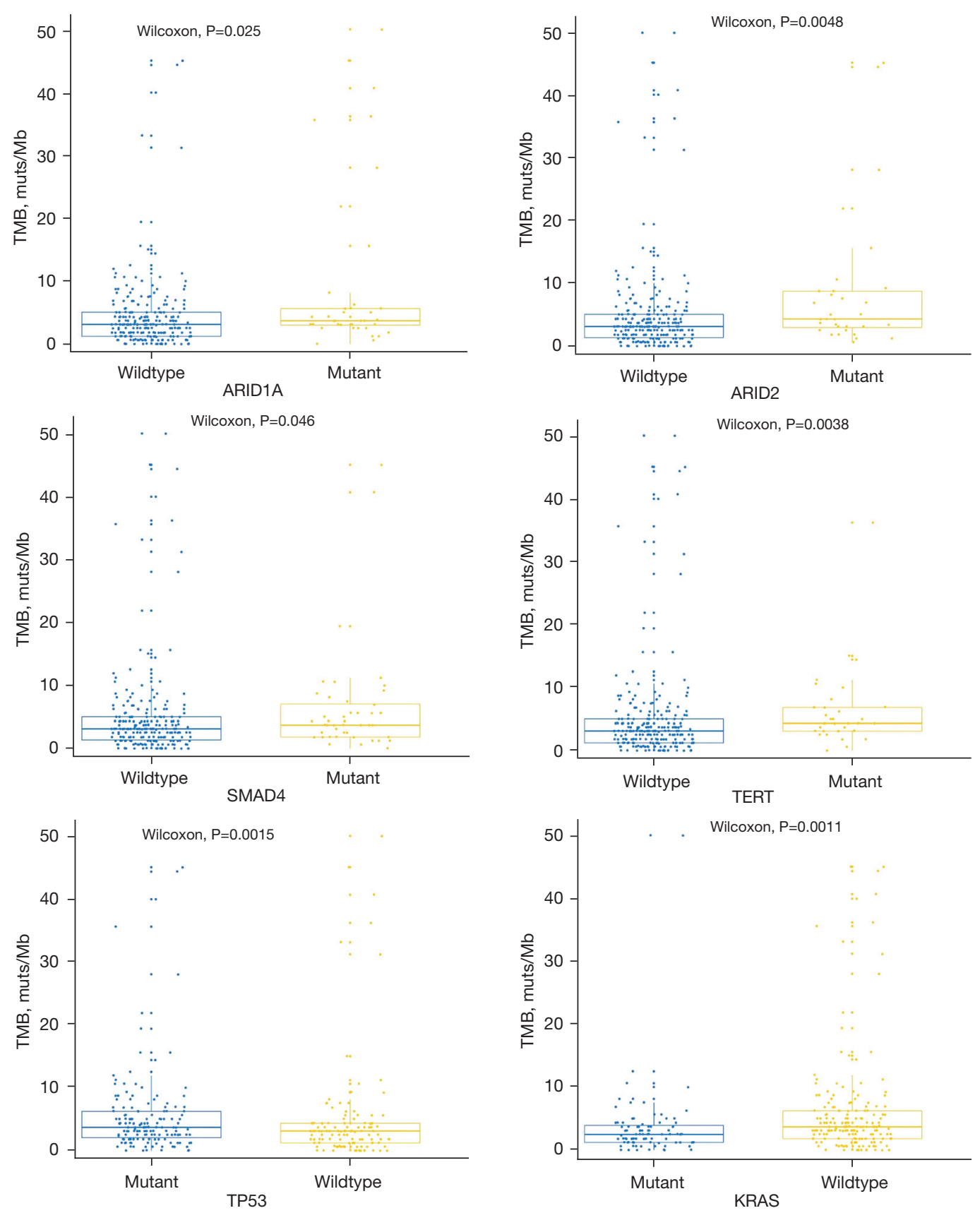

Figure 3 The association between TMB value and ARID1A, ARID2, SMAD4, TERT, TP53, and KRAS mutations. TMB, tumor mutational burden.

development of NGS detection technology, numerous studies have investigated the molecular characteristics of CCA subtypes. Similar to previous studies $(14,15)$, we found that the IDH1, BAP1 and FGFR2 mutations were most common in iCCA, and TP53, SMAD4, and ERBB2 mutations were most common in exCCA. These findings indicate that the profiling of CCA is similar between Chinese patients and Western patients. Available drug targets in CCA include FGFR2 and IDH1. Our results supported that patients with iCCA have more opportunity to benefit from targeted therapy than those with exCCA.

In this study, we distinguished pCCA from dCCA 
A

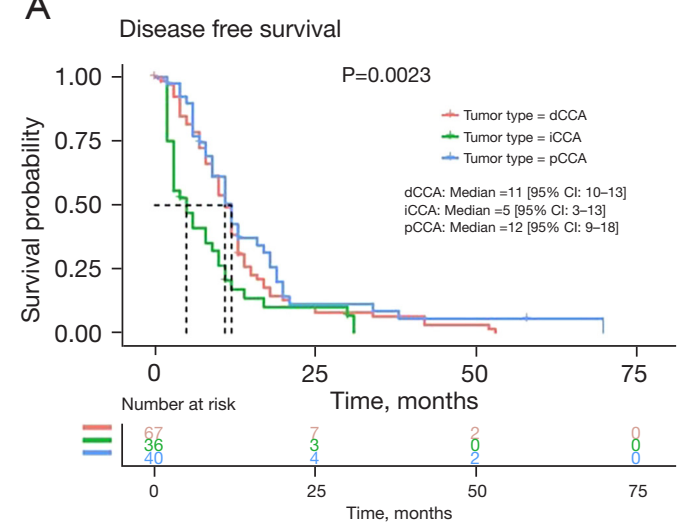

B

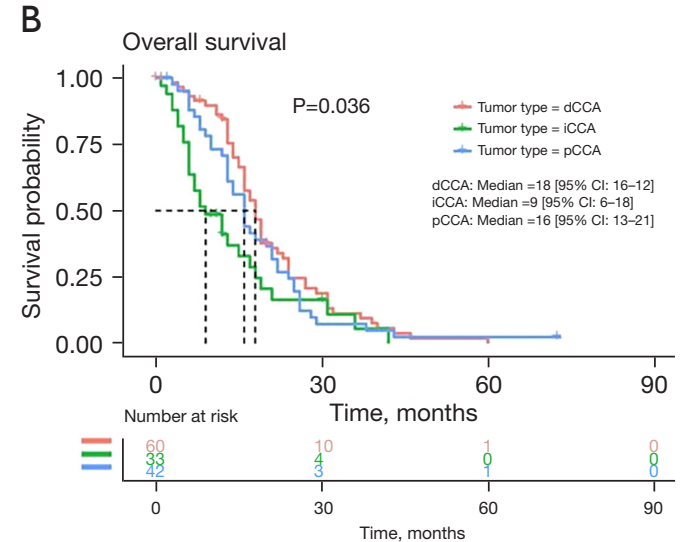

Figure 4 Analysis of disease-free survival (A) and overall survival (B) of patients with different CCA subtypes. CCA, cholangiocarcinoma.

in exCCA by identifying the characteristic molecular alterations between them. Our results showed more frequent mutations of TP53 and KRAS in dCCA, and FAT4, KDM6A, MDM2, and TCF7L2 in pCCA. Although pCCA is classified as exCCA, its location is closer to iCCA, which makes it difficult to classify accurately to some extent. In addition to the specific frequently occurring FAT4, KDM6A, and $M D M 2$ mutations in pCCA, the mutational frequencies of SMAD4, ARID2, ARID1A, and ERBB2 were similar to dCCA, while the mutational frequencies of TERT and PIK3CA were similar to iCCA. These results highlight the complex mutation characteristics of pCCA. Although CCA subtypes were not distinguished at the molecular level yet, it will greatly promote the development of CCA precision medicine in the future.

Based on prognosis analysis, we also identified different potential biomarkers in both pCCA and dCCA. Interestingly, our results showed that $N F 1$ mutation was associated with poor prognosis in dCCA, while MAP2K4 mutation was associated with better prognosis in pCCA. NF1 is reported as a tumor suppressor gene and is associated with poor prognosis in tumors (16). MAP2K2 is involved in multiple cellular processes, including cell differentiation, apoptosis, and proliferation (17). High expression of $M A P 2 K 2$ is reportedly associated with poor OS in breast cancer (18), implying an association between $M A P 2 K 2$ mutation and good prognosis. Together with our results, we therefore conclude the potential predictive role of NF1 and MAP2K2 in the prognosis of dCCA and pCCA, respectively. These results also illustrated the different molecular characterization between pCCA and dCCA.

In addition, we also detected the association between ERBB2 mutation and DFS in dCCA, and the association between TERT mutation and DFS in pCCA. Previous studies have reported the association between TERT and $E R B B 2$ and prognosis in non-small cell lung cancer and CCA $(14,19)$. This supported that both ERBB2 and TERT could be potential biomarkers for prognosis prediction. More importantly, our results emphasized that the correlation exists only in one subtype of exCCA, which demonstrates the importance of accurate classification for further treatment and prognosis prediction, and also supported the necessity of NGS detection for precision treatment of CCA.

KRAS mutational frequency is different between iCCA and exCCA (20), and is greatly valued for improving the prognosis of iCCA (21). RBM10 has been reported to regulate the Notch pathway by interacting with $N U M B$ in cancer (22), and the Notch pathway can predict the prognosis of many cancers. In this study, we identified an association between KRAS and RBM10 and DFS or OS in iCCA, which supported that KRAS and RBM10 mutation may be potential biomarkers for prognosis prediction in iCCA. In this study, nearly $10 \%$ of FGFR2 fusions/ rearrangements were detected in iCCA, but we failed to identify an association between FGFR2 fusion and DFS in iCCA. This is inconsistent with previously reported association between FGFR 2 fusion and improved OS in iCCA (23). We deduced that the small number of patients or the limited follow-up data may be potential limitations in this study, or may be due to regional differences. However, this requires confirmation by further research.

TMB reflects the number of somatic mutations in the genome sequence. Tumors with TMB-H are well recognized as having more non-selfantigens or neoantigens, to be potentially recognized by the host immune 

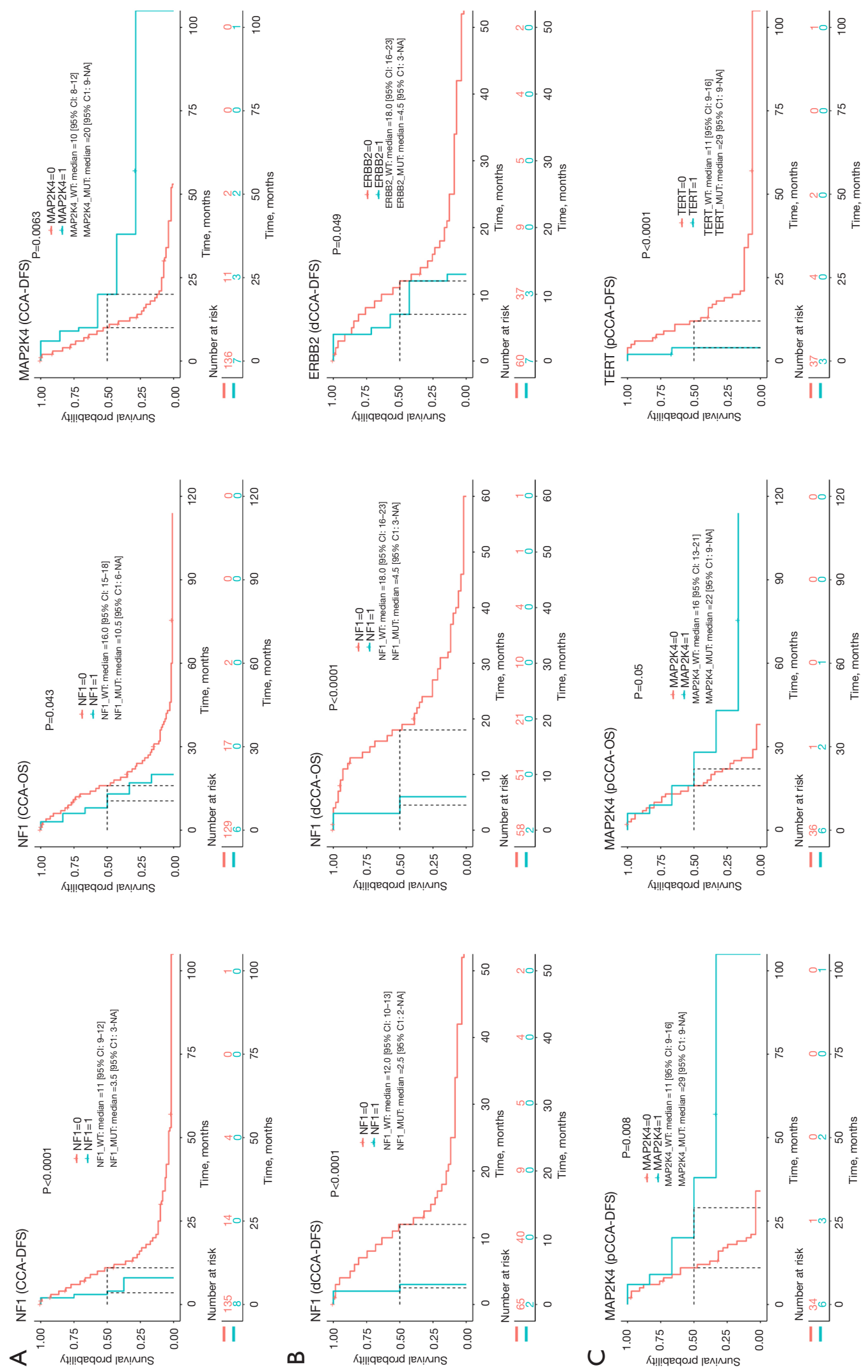

(C) Journal of Gastrointestinal Oncology. All rights reserved. $\quad 7$ Gastrointest Oncol 2021;12(6):2631-2642 । https://dx.doi.org/10.21037/jgo-21-776 

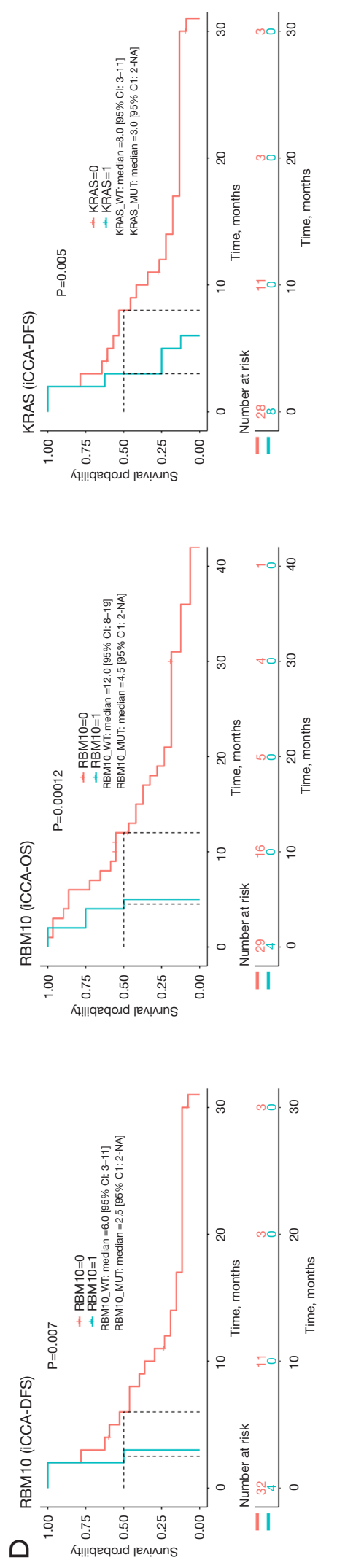

system more frequently. TMB is an effective biomarker that can further guide patients to choose checkpoint inhibitors (24). Different cancer types have different distributions of TMB values; however, TMB-H is associated with improved survival in most patients who receive immune checkpoint inhibitors treatment. Previous study showed that the median TMB of CCA was only 1.23 mutations/Mb (25). Zhang et al. showed CCA patients (one iCCA and two dCCAs) with TMB-H well benefited from immune checkpoint inhibitors (26). Weinberg et al. reported that TMB in iCCA was higher than that in exCCA (27). In this study, we did not find a significant difference in the TMB between CCA subtypes. Therefore, we analyzed the TMB-related gene mutations in the whole CCA cohort. Our results showed that ARID1A, ARID2, SMAD4, TERT, and TP53 mutations were associated with TMB-H, while the KRAS mutations were associated with TMB-L, which implied a potential opportunity for CCA patients with/without these mutations.

Numerous studies have demonstrated the association between ARID1A mutation and poor prognosis in many cancer types (28). High SMAD4 levels can predict a better prognosis in colorectal cancer (29), while the loss of SMAD4 mutations is associated with poor prognosis in colorectal cancer (30). TERT and TP53 mutations are reportedly associated with poor prognosis in many cancer types (19,31). Moreover, KRAS mutation is reportedly associated with poor prognosis in patients with different cancer types (32). In this study, except for the association between KRAS mutation and poor prognosis in iCCA, we did not identify any associations between the mutations of these genes and prognosis, which suggests that predicting prognosis using TMB levels in CCA would be difficult.

Surgery is the preferred treatment option for CCA patients. However, the prognosis is different among CCA subtypes. Based on a study of 564 patients with R0resections, the median survivals of iCCA, pCCA, and dCCA were 80,30 , and 25 months, respectively (33). Although we selected patients with early tumor stage (stage I/II) for prognosis analysis, the results showed that the prognosis of iCCA was worse than that of exCCA, and there was no significant difference in the prognosis between pCCA and dCCA. This may be largely affected by tumor stage. Most patients in our study were diagnosed early, and postoperative pathology showed a higher proportion of advancement in iCCA compared to exCCA. This implies that early iCCA has the potential to progress faster. In addition, patient survival rates may vary by country or 
region. Anyway, with the development of tumor precision medicine, clinical studies of combined therapy based on NGS technology are expected to improve the survival of CCA patients.

In summary, we investigated the comprehensive genomic characterizations of $270 \mathrm{CCA}$ patients and identified the significant alterations in each subtype. These results suggest different molecular features between pCCA and dCCA. Furthermore, prognosis analysis identified potential biomarkers for prognosis prediction, such as MAP2K4 mutations in pCCA and NF1 in dCCA. Together, our study provides evidence for further precision targeted therapy of CCA patients.

\section{Acknowledgments}

Funding: This work was supported by the Science and technology development project of Jinan City (201907073), the Key R\&D project of Shandong Province (2019GSF108231), and the Key R\&D project of Shandong Province (2016GGB14064).

\section{Footnote}

Reporting Checklist: The authors have completed the REMARK reporting checklist. Available at https://dx.doi. org/10.21037/jgo-21-776

Data Sharing Statement: Available at https://dx.doi. org/10.21037/jgo-21-776

Conflicts of Interest: All authors have completed the ICMJE uniform disclosure form (available at https://dx.doi. org/10.21037/jgo-21-776). Dr. JS, Dr. XS and Dr. KW report that they serve as employees of OrigiMed Co. Ltd, Shanghai, China. The other authors have no conflicts of interest to declare.

Ethical Statement: The authors are accountable for all aspects of the work in ensuring that questions related to the accuracy or integrity of any part of the work are appropriately investigated and resolved. The study was conducted according to the guidelines of the Declaration of Helsinki (as revised in 2013), and it was approved by the Institutional Ethics Review Committee at Shandong Provincial Hospital (ethics approval number: LCYJ: No. 2019-081). Informed consent was obtained from all subjects involved in the study.
Open Access Statement: This is an Open Access article distributed in accordance with the Creative Commons Attribution-NonCommercial-NoDerivs 4.0 International License (CC BY-NC-ND 4.0), which permits the noncommercial replication and distribution of the article with the strict proviso that no changes or edits are made and the original work is properly cited (including links to both the formal publication through the relevant DOI and the license). See: https://creativecommons.org/licenses/by-nc-nd/4.0/.

\section{References}

1. Hezel AF, Zhu AX. Systemic therapy for biliary tract cancers. Oncologist 2008;13:415-23.

2. Marcano-Bonilla L, Mohamed EA, Mounajjed T, et al. Biliary tract cancers: epidemiology, molecular pathogenesis and genetic risk associations. Chin Clin Oncol 2016;5:61.

3. Clements O, Eliahoo J, Kim JU, et al. Risk factors for intrahepatic and extrahepatic cholangiocarcinoma: A systematic review and meta-analysis. J Hepatol 2020;72:95-103.

4. Brandi G. Hot topics in cholangiocarcinoma. Transl Cancer Res 2019;8:S219-22.

5. Chan KM, Tsai CY, Yeh CN, et al. Characterization of intrahepatic cholangiocarcinoma after curative resection: outcome, prognostic factor, and recurrence. BMC Gastroenterol 2018;18:180.

6. Goldaracena N, Gorgen A, Sapisochin G. Current status of liver transplantation for cholangiocarcinoma. Liver Transpl 2018;24:294-303.

7. Kobayashi S, Terashima T, Shiba S, et al. Multicenter retrospective analysis of systemic chemotherapy for unresectable combined hepatocellular and cholangiocarcinoma. Cancer Sci 2018;109:2549-57.

8. Valle J, Wasan H, Palmer DH, et al. Cisplatin plus gemcitabine versus gemcitabine for biliary tract cancer. $\mathrm{N}$ Engl J Med 2010;362:1273-81.

9. Simile MM, Bagella P, Vidili G, et al. Targeted Therapies in Cholangiocarcinoma: Emerging Evidence from Clinical Trials. Medicina (Kaunas) 2019;55:42.

10. Nakamura H, Arai Y, Totoki Y, et al. Genomic spectra of biliary tract cancer. Nat Genet 2015;47:1003-10.

11. Tian W, Hu W, Shi X, et al. Comprehensive genomic profile of cholangiocarcinomas in China. Oncol Lett 2020;19:3101-10.

12. Feng F, Wu X, Shi X, et al. Comprehensive analysis of genomic alterations of Chinese hilar cholangiocarcinoma patients. Int J Clin Oncol 2021;26:717-27. 
13. Waseem D, Tushar P. Intrahepatic, perihilar and distal cholangiocarcinoma: Management and outcomes. Ann Hepatol 2017;16:133-9.

14. Lowery MA, Ptashkin R, Jordan E, et al. Comprehensive Molecular Profiling of Intrahepatic and Extrahepatic Cholangiocarcinomas: Potential Targets for Intervention. Clin Cancer Res 2018;24:4154-61.

15. Xue L, Guo C, Zhang K, et al. Comprehensive molecular profiling of extrahepatic cholangiocarcinoma in Chinese population and potential targets for clinical practice. Hepatobiliary Surg Nutr 2019;8:615-22.

16. Mei Z, Shao YW, Lin P, et al. SMAD4 and NF1 mutations as potential biomarkers for poor prognosis to cetuximabbased therapy in Chinese metastatic colorectal cancer patients. BMC Cancer 2018;18:479.

17. Roskoski R Jr. MEK1/2 dual-specificity protein kinases: structure and regulation. Biochem Biophys Res Commun 2012;417:5-10.

18. Yu S, Zhang M, Huang L, et al. ERK1 indicates good prognosis and inhibits breast cancer progression by suppressing YAP1 signaling. Aging (Albany NY) 2019;11:12295-314.

19. Jung SJ, Kim DS, Park WJ, et al. Mutation of the TERT promoter leads to poor prognosis of patients with nonsmall cell lung cancer. Oncol Lett 2017;14:1609-14.

20. Andersen JB, Spee B, Blechacz BR, et al. Genomic and genetic characterization of cholangiocarcinoma identifies therapeutic targets for tyrosine kinase inhibitors. Gastroenterology 2012;142:1021-1031.e15.

21. Robertson S, Hyder O, Dodson R, et al. The frequency of KRAS and BRAF mutations in intrahepatic cholangiocarcinomas and their correlation with clinical outcome. Hum Pathol 2013;44:2768-73.

22. Hernández J, Bechara E, Schlesinger D, et al. Tumor suppressor properties of the splicing regulatory factor RBM10. RNA Biol 2016;13:466-72.

23. Graham RP, Barr Fritcher EG, Pestova E, et al. Fibroblast growth factor receptor 2 translocations in intrahepatic cholangiocarcinoma. Hum Pathol 2014;45:1630-8.

Cite this article as: Zheng Y, Qin Y, Gong W, Li H, Li B, Wang Y, Chao B, Zhao S, Liu L, Yao S, Shi J, Shi X, Wang K, $\mathrm{Xu}$ S. Specific genomic alterations and prognostic analysis of perihilar cholangiocarcinoma and distal cholangiocarcinoma. J Gastrointest Oncol 2021;12(6):2631-2642. doi: 10.21037/jgo21-776
24. Wu HX, Wang ZX, Zhao Q, et al. Tumor mutational and indel burden: a systematic pan-cancer evaluation as prognostic biomarkers. Ann Transl Med 2019;7:640.

25. Lin J, Cao Y, Yang X, et al. Mutational spectrum and precision oncology for biliary tract carcinoma. Theranostics 2021;11:4585-98.

26. Zhang W, Shi J, Wang Y, et al. Next-generation sequencing-guided molecular-targeted therapy and immunotherapy for biliary tract cancers. Cancer Immunol Immunother 2021;70:1001-14.

27. Weinberg BA, Xiu J, Lindberg MR, et al. Molecular profiling of biliary cancers reveals distinct molecular alterations and potential therapeutic targets. J Gastrointest Oncol 2019;10:652-62.

28. Bi C, Liu M, Rong W, et al. High Beclin-1 and ARID1A expression corelates with poor survival and high recurrence in intrahepatic cholangiocarcinoma: a histopathological retrospective study. BMC Cancer 2019;19:213.

29. Isaksson-Mettävainio M, Palmqvist R, Dahlin AM, et al. High SMAD4 levels appear in microsatellite instability and hypermethylated colon cancers, and indicate a better prognosis. Int J Cancer 2012;131:779-88.

30. Oyanagi H, Shimada Y, Nagahashi M, et al. SMAD4 alteration associates with invasive-front pathological markers and poor prognosis in colorectal cancer. Histopathology 2019;74:873-82.

31. Shen N, Lu Y, Wang X, et al. Association between rs2853669 in TERT gene and the risk and prognosis of human cancer: a systematic review and meta-analysis. Oncotarget 2017;8:50864-72.

32. Mehrad M, Roy S, LaFramboise WA, et al. KRAS mutation is predictive of outcome in patients with pulmonary sarcomatoid carcinoma. Histopathology 2018;73:207-14.

33. DeOliveira ML, Cunningham SC, Cameron JL, et al. Cholangiocarcinoma: thirty-one-year experience with 564 patients at a single institution. Ann Surg 2007;245:755-62.

(English Language Editor: A. Kassem) 



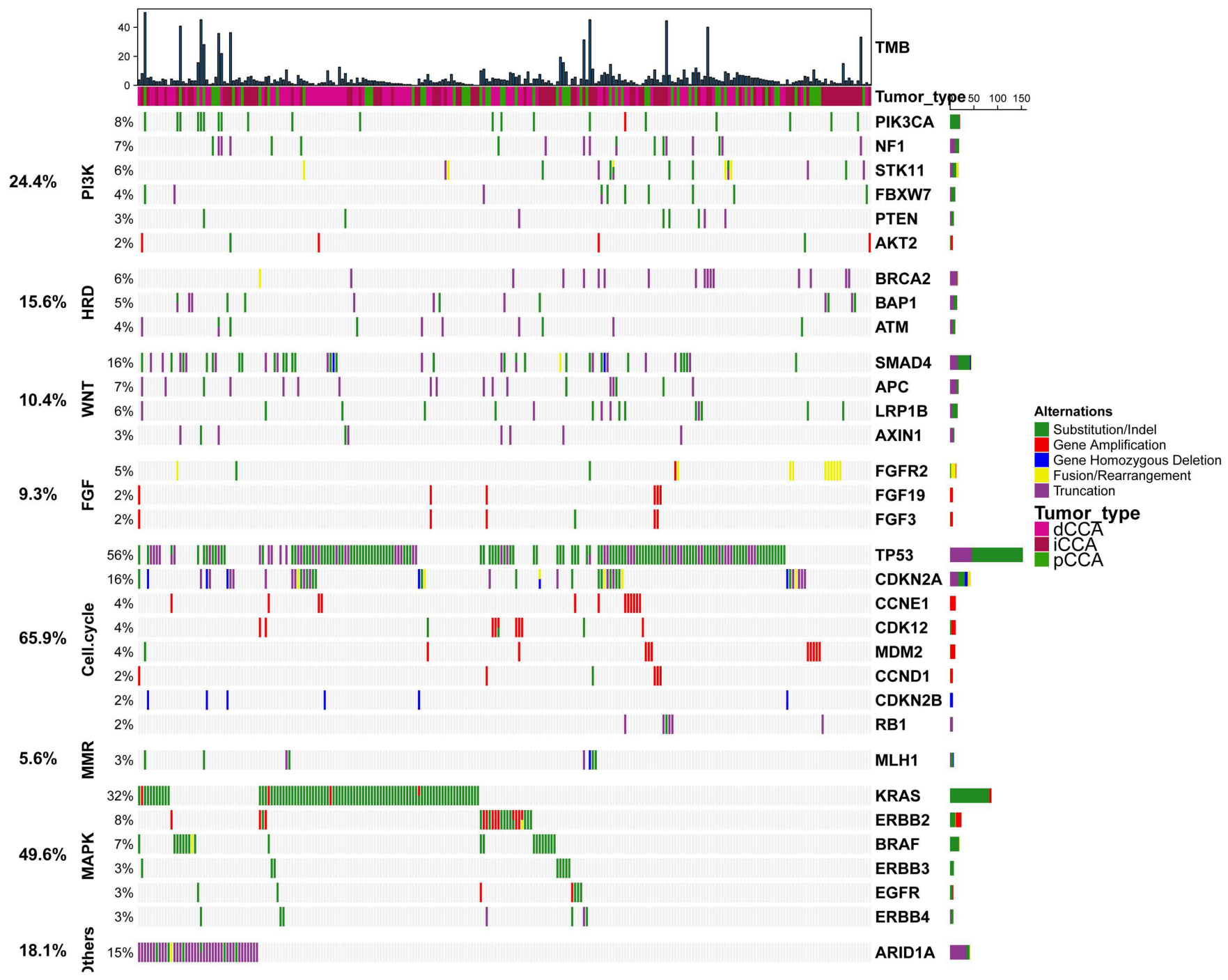

Figure S1 Pathway mutational landscape of 270 CCAs. CCA, cholangiocarcinoma. 


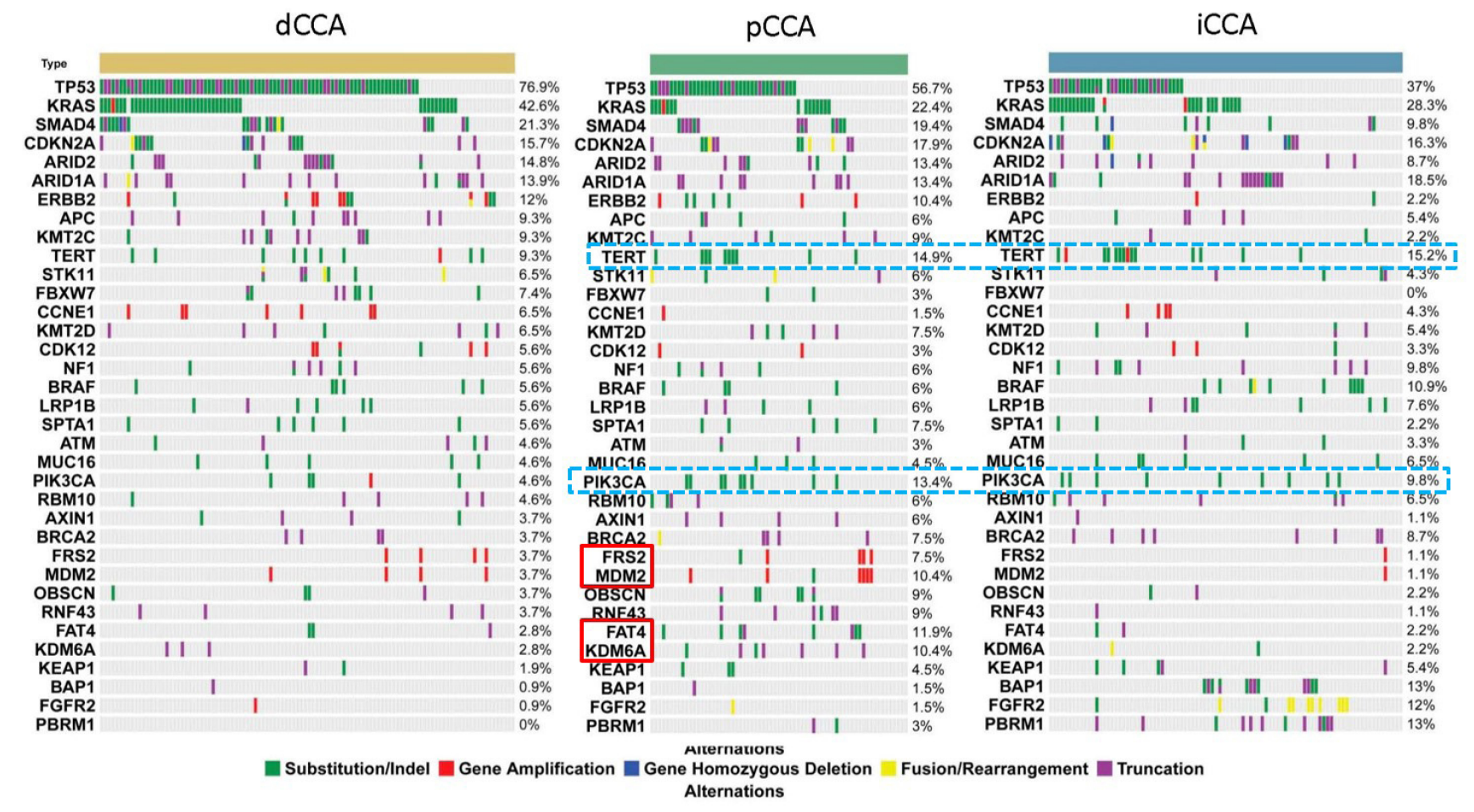

Figure S2 The most commonly mutated genes (>10\%) in different CCA subtypes. CCA, cholangiocarcinoma. 